\title{
Design Implications for a Community-based Social Recipe System
}

\author{
Veranika Lim, Fulya Yalvaç, \\ Mathias Funk, Jun Hu and \\ Matthias Rauterberg \\ Designed Intelligence Group \\ Department of Industrial Design \\ Eindhoven University of Technology \\ The Netherlands
}

\author{
Carlo Regazzoni \\ and Lucio Marcenaro \\ Department of Electrical \\ Electronic, Telecommunications \\ Engineering and Naval Architecture \\ University of Genoa \\ Italy
}

\begin{abstract}
We introduced the concept of a community-based social recipe system which suggests recipes to groups of users based on available ingredients from these users (i.e. who can be from the same household or different households). In this paper we discuss the relevance and desirability of such a system and how it should be designed based on user studies. We identified the relevance of targeting ingredients and found positive expected experiences with the system such as to prevent habitual waste-related behavior, awareness of in-home food availability, creativity in cooking, moments for surprises and spontaneity, coordination among a group of friends, education and connectedness. Possible reasons of not using the system are trust and the inconvenience of distance among users in a group that are suggested with a social recipe. From our findings, we specify design implications for the system and optimization functions aiming at the prevention of food waste at a collective level.
\end{abstract}

\section{INTRODUCTION}

Food waste is a complex global issue with impacts on the environment and food security. In developed countries, roughly half of the total avoidable losses within the food chain is generated by consumers [1] which has resulted into prospects for redirecting consumer consumption patterns towards sustainable practices to reduce environmental impacts [2]. Preventing or reducing food waste generated by consumers, however, is considered a major challenge as many factors are involved. These factors are, for example, knowledge [3][4], skills and planning with regard to preparation and cooking practices [5][6]. Other factors, such as memory, attitude [5][6] and general beliefs together with education and political affiliation, were also found to be stable predictors of overall environmental concern [7]. Having busy lifestyles, social relations and the unpredictability of events are other important factors [5][6]. Moreover, our everyday behaviors around food have become less conscious and decisions resulting in food waste are often implicit, indirectly linked or hidden [5][6]. Therefore, it is important to raise awareness of food waste patterns and design intelligent solutions that are embedded and accepted in our daily lives that motivate people to reduce and avoid wasteful behaviors.
In the field of Human Computer Interaction (HCI), recent research suggests exploring the roles of collectivism and community for food sharing practices as a way to reduce food waste [8][9]. Related to these findings, we presented Euphoria, a project working towards the design of a community-based social recipe system [10]. In this concept, ingredients available from different households are combined into one or more recipes, which are suggested to a group of users with the main aim at collective food waste prevention through collaboration and food sharing. Apart from its altruistic aim, this approach incentivizes people to share, cook and enjoy food together. In this study we explore the relevance and desirability from the user perspective, contributing to the design of the system and its food waste prevention potential. Including user studies early in the design process is expected to result in more relevant specifications of the behavior of the system and hence, increases the likeliness of acceptance in daily lives. The objective of this study is threefold: first, to identify amounts and types of food waste as well as the reason of wastage which would provide a basis of the proposed system. Second, to explore users' expected experiences of a community-based social recipe system. Finally, to relate findings with design implications for the behavior of the system in optimizing food waste by means of recipe suggestions.

\section{RELATED WORK}

In $\mathrm{HCI}$, persuasive sustainability research is increasing in popularity. It has, however, mainly focused on issues such as energy consumption, water consumption or green transportation with the aim to increase awareness [11]. Ecofeedback is an example of a strategy to increase awareness of resource use and encourage conservation by automatically sensing people's activities and feeding related information back through computerized means [12]. It aims at fostering positive attitudes towards sustainable practices aiming at conservation [13]. Some examples of Eco-feedback displays are described in [14] and [15]. Eco-feedback, however, does not necessarily direct behavior change explicitly. With our system, we are interested in the possibilities beyond attitude change (i.e. behavior change). Maitland et al. [16] suggest that for 
persuasive technology to be successful, it should be designed to encourage action. Systems designed for action was argued to have impacts on creativity, pleasure and nostalgia, gifting, connectedness and trend-seeking behaviors [16]. Encouraging collective action is a major characteristic of Euphoria [10].

Next to designing for action, social influence strategies have also been found to have high potential as a means for generating positive behavior change [17]. Social mechanisms that humans use to influence others, such as social approval, peer pressure, norm activation or social comparison, are principles that can be applied successfully for supporting behavior change [18]. The use of social influence strategies is another major characteristic of Euphoria [10]. Studies have shown that our social environments are important determinants of food waste related behavior [5][6], stemming from cultural practices, signaling social status, but also emerging behavior in an age of abundant choice and quantity of food. The social context, therefore, plays a major role in shaping our individual decision-making processes, specifically in the area of food waste. This highlights the importance of addressing the collective as a target for behavior change as suggested in [9]. In fact, in previous findings, social activity was found to be a determinant of food waste [6]. With a communitybased social recipe system, we aim at using social activity to discourage food waste, which is in accordance with the celebratory technology described in [19]. Several examples of technologies exist in the area of human-food interaction that celebrates the positive relationships people have with food in their everyday social lives. One such example is Foodmunity [20], a platform for the community through which members can share personal experiences about meals. The main aim of the platform is to share these experiences with others as a basis for exposing people to the new and the unknown. Another example is a food recipe system called Kalas [21]. This system, which includes aggregated trails of user actions, provides different means of communication between users. EatWell [22] is a system for sharing nutrition-related memories targeting low-income communities. The system allows people to use their cell phones to create voice memories describing how they have tried to eat healthfully in their neighborhoods (e.g., at local restaurants) and listen to the memories that others have created. Barden et al. [23] designed a technology platform that supports remote guests in experiencing togetherness and playfulness within the practices of a traditional dinner party. Furthermore, in [24], a menu-planning support system is presented to facilitate interaction and communication among neighbors. Their system allows users to manually select their preferences of food and neighbors. This information is later used to propose dishes consisting of shared ingredients owned by a number of individuals.

Although these projects study food-related practices on a collective level, they do not explore sustainable food-related decision-making specifically such as the influence on food waste. Currently, we are only aware of the work described in [25], where sustainable food-related decision-making was explored to understand issues of sharing and the use of social networking in an activist food sharing community. With Euphoria we are interested in how the concept of social recipes influence social dynamics around food related practices and its advantages on food waste, an important topic for sustainability.

\section{EUPHORIA}

Euphoria (Efficient food Use and food waste Prevention in Households through Increased Awareness) allows users to $\log$ and track available in-home ingredients as well as their wasteful behaviors. Based on this information, the system would help users to redirect behaviors, through social influence, towards more sustainable food related practices in terms of food waste. The main function of the system is to detect potential food waste and respond by providing social recipes before the food get wasted. Social recipes contain available ingredients from different households that need to be consumed in time. In this sense, it would target prevention at the collective community level. The promotion of social interaction is expected to gain more effective food waste prevention as it provides a new pleasurable experience around food practices. The next section is to clarify our current development progress and how the system will be tested and deployed in future work.

\section{A. Apparatus}

For the logging and tracking of in-home ingredients and wasteful behaviors, we have developed a mobile application for iOS and Android with a hybrid approach using PhoneGap (See Figure 1). At the first log in, users can set their user profiles including their demographics. On a daily basis, users can search and select ingredients and move it to their wish list or stock list. In these lists, users can indicate the amounts (in weights, numbers or liters), move items from their wish list to their stock list when an item is bought and indicate consumption in the stock list. Whenever an item is wasted users can select the reasons of disposal. A survey is integrated in the application to measure the perception of control in wasteful behaviors.

We used JQueryMobile, HTML and CSS for the user interface of the mobile application and JavaScript for the user interaction. The server side was developed with the PLAY framework for JAVA. The data flow between the client and the server is carried with JSON objects. Data from the users are stored in the local database of the smart phones by using an SQLite database engine and sent to the server database which is provided by the PLAY framework (when the smart phone is connected to the internet).

The mobile application can be used in two ways: to provide the social recipe module with the available ingredients that might be likely to get wasted and when a social recipe is provided, it can be used to see whether there are changes in available items or wasteful behaviors. This would allow 

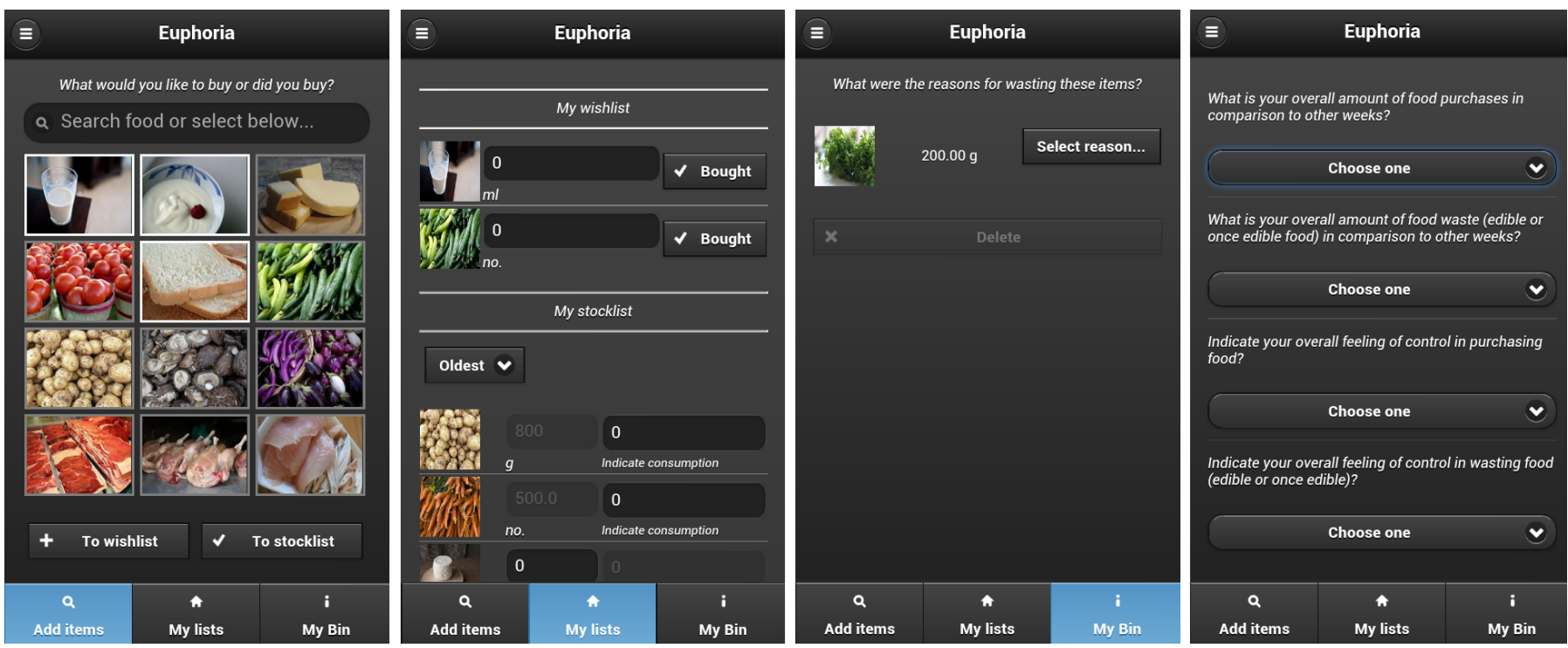

Fig. 1. Interface design of the mobile application.

us to evaluate the system. Before integrating the module and user interface for social recipe suggestions in the mobile application, we first need to explore how to design the behavior of the system which is the main contribution of this paper. In the next section, our user-centered approach, findings and its implications on the design of the community-based social recipe system are explained.

\section{USER STUDIES}

\section{A. Exploratory field study}

The exploratory field study took place from mid-February to mid-April 2014 with participants living in urban areas in the Netherlands. We had the following objectives:

- Identify amounts and types of food waste as well as the reasons of wastage.

- Explore users' expected experiences of a communitybased social recipe system.

- Relate findings with design implications for the behavior of the system in optimizing food waste by means of recipe suggestions.

1) Methodology: At the beginning of the study, we asked participants to collect their grocery receipts that were later used as cues for biweekly retrospective interviews on their food wastage during the past weeks. Participants received a small box to store the receipts and a black marker to cover any item that was considered private. They were further provided with a table bin and a kitchen scale and were asked to weigh the waste every time before emptying the bin and to write down the grams on a log sheet. The log sheet was replaced after each interview. Participants were also asked to separate organic waste from other generated waste (e.g. plastics, paper etc.) and were instructed to include all edible as well as nonedible parts of food items such as bones, tea bags, egg shells and banana peels. This was done to prevent differences in the definition of edibility. Participants were interviewed twice, individually, in couples or in the presence of other group members, depending on their living circumstances. Overall, we aimed at familiarizing with users' reasons of waste and social practices around food such as shared activities in shopping, paying for shared groceries, cooking and eating. During the last interviews, a description of Euphoria was explained to users verbally in a hypothetical fashion to gather their expected experiences and initial ideas about the concept.

2) Participant demographics: The study was carried out with 28 national and international students and young professionals in the age range of 22 to 31. Participants were subdivided into 8 groups based on different levels of proximity, i.e. living together and sharing the same kitchen, living in the same complex or living in the same city. We did not specify any requirements on the participation other than being a student or a young professional living in urban areas. Participants were recruited through social and personal networks and were visited at their homes after work hours by the same researcher and were compensated by means of vouchers. The following provides a description of each group of participants.

Group A; consisted of 4 international students living in single studios on campus. 3 students were from India and 1 from China. They all mainly cook for themselves during the week for two to four days.

Group B; consisted of 3 international students living in an apartment with a shared kitchen. 2 students were from Portugal and always do groceries and dinners together. The other student from Germany mainly cooks for herself as she is a vegetarian unlike the others. 
Group C; consisted of 2 international couples of young professionals, each living in different apartments, but in the same city. One couple came from Turkey and the other couple from Australia and Turkey respectively. The first couple is married and all are friends of each other.

Group D; consisted of 5 international students from India. All are friends of each other but are living in different houses. Two live in a studio, one with 2 Dutch students in an apartment with a shared kitchen and the last two live together in an international house for 7 students with one shared kitchen.

Group E; are good friends living in a house with a shared kitchen. One is doing a $\mathrm{PhD}$ while the others were graduating. They are Dutch, have similar friends and travel together regularly.

Group F; consisted of 2 Dutch young professionals who are living together with a shared kitchen. They describe themselves as very busy, which was given as a reason for not cooking and eating home often.

Group G; consisted of 3 Dutch female students who are living together in a student house with a shared kitchen. They are good friends and are part of a sorority club. Overall, they are socially very active and have dinners in big groups at least once a week.

Group H; consisted of 5 Dutch female students who are also living together in a student house with a shared kitchen. All are members of a sports club and are also socially very active. They cook and eat together regularly.

\section{B. Focus group}

In addition to the exploratory field study and as a follow up, a double-blind focus group study was conducted with six $\mathrm{PhD}$ students and one moderator. A limitation of the exploratory field study is the awareness of the purpose of the study is that participants were aware of the purpose of the study, which could have had an effect on their overall behavior and comments. We were interested to see how participants would respond to the system without immediately relating it to the negative behavior around food waste. To keep the moderator in a neutral position, a double-blind procedure was used to guard against experimenter bias and influences.

1) Methodology: After some warm-up questions about food experiences in general, participants were asked about food items they had available at home. They were also asked whether they would want to exchange these items with others and/or to combine it with other peoples' food items into a meal. Next, the concept was presented with the following description of the social recipe recommendation system:

'Imagine a system that knows which foods you have in your house, which foods your friends have in their homes, and that can suggest you to get together with your friends to make a recipe with the available ingredients without having to go to the grocery store.'

Participants were then asked several questions regarding how the system would affect them, who they would like to use this system with, and how they envision this system would affect their group of friends. Two researchers attended the sessions for observation and the sessions were video recorded.

2) Participant demographics: Participants for this study were recruited based on several requirements: first, they all had to live with at least another person at home. Second, they had to cook at home at least three times a week. Third, they should be eating and cooking with friends at least twice a week and finally, they had to do groceries themselves. The students were all from China living in the Netherlands, and they were compensated with lunch. Our choice for selecting Chinese students is because of their cooking culture; they cook regularly in social settings. Although, much less food is wasted at the consumer level in non-Western countries (low-income) [1], as the world largest emerging economy, China is starting to suffer a high wastage of food during consumption [26]. In the next section, we will mainly discuss findings from the exploratory field study unless indicated otherwise (i.e. from the focus group).

\section{STUDY FINDINGS}

A total of 231 food items were wasted over the whole study period excluding drinks (other than milk), desserts, cookies, and confectioneries. A food item was defined as equal to a single fruit or vegetable such as one banana or one cabbage, a basket of smaller fruits or vegetables such as cherry tomatoes or grapes, or one portion of rice or pasta. Each reported food item was further categorized into different food groups: fruits, grains, dairy, vegetables, meat and fish, or other (e.g. sandwich spreads). Almost half of all the wasted items were vegetables. These vegetables were wasted partly with an average of 64 percent of the whole item. This finding supports the choice of targeting ingredients, specifically perishables.

\section{A. Food group in relation to the reason of wasting}

We used thematic analysis to categorize the reasons that were provided for wasting:

- Way of consumption; includes items that were used only for flavoring or parts were cut away because of the recipe.

- Items gone badly; includes all items with visual characteristics of decay such as mold, decoloration, or growths through the skins, for example, in potatoes. These could further be caused by forgetfulness, busy lives, too big purchases, unpredictability of longevity, change of meal plans, the weather, etc.

- Doubtful items; includes visual unattractiveness such as drought or over-moisture, expiration dates, items that were left open in the kitchen for one or several days and 
were not trusted anymore in terms of quality, and items that were just considered old and had been in the fridge for a long time. These could also further be caused by forgetfulness, busy lives, social activities or knowledge.

- Dealing with leftovers; includes cooked or prepared ingredients that were left after dinner but not worthwhile saving (e.g. too little to save or not tasting good) or leftovers without plans for being used in the near future. This category also includes meals that were saved for several days with the intention of usage but were eventually forgotten (cf. causes above).

- Other; other reasons include the way of saving items (e.g. without foil), food items that were partly bad at the time of purchase, unexpected taste of items, difficulty in getting it out of a package or simply just due to a bad fridge or not using a non-stick pan.

Vegetables were found to be wasted due to physical deterioration $(\mathrm{N}=38)$ or were expected not to be edible and thus doubtful in quality and safety $(\mathrm{N}=41)$. Hence, this also supports the potential of targeting vegetables with the social recipe system.

1) Implications on the system design: the use of existing ingredients to suggest social recipes with the intention of reducing food waste can be defined and explored as a constraint satisfaction problem. The social recipe system should find optimum recipes that can save food with the potential of being wasted. Therefore, it should consider minimizing the amount of available ingredients as the most important constraint.

The first implication is on prioritizing ingredients. As users can enter food items in the mobile application, the system will know the type of item, its amount, when the item is added and how long the item has been in the stock list (availability). By comparing the duration of availability with the average longevity of the specific food item (which can be derived from a database), different risk levels can be assigned. We will distinguish three levels of risk: high (the item is good for max. 2 days), medium (the item is good for max. 4 days) or low (the item is good for more than 4 days). Optimization functions can be defined as:

$$
\begin{aligned}
& \operatorname{minimize} \sum_{i}^{L H} \sum_{j}^{U} \operatorname{Amount}(i, j) \\
& \operatorname{minimize} \sum_{i}^{L M} \sum_{j}^{U} \operatorname{Amount}(i, j)
\end{aligned}
$$

where:

- $L H$ : are items with high risk of being wasted.

- $L M$ : are items with medium risk of being wasted.

- $U$ : is the list of users receiving the recipe suggestion.

- Amount $(i, j)$ : is the amount of item $i$, user $j$ has.

Equation (1) will have the highest weight in the overall constraint model while the low risk values will not be taken into account as a constraint.
A second implication is to match available ingredients with ingredients necessary for a specific recipe. In most of the cases, the amount of the available ingredients do not exactly match with recipe requirements so in real life, people would probably modify the recipe according to the ingredients they have. The system should be specified with a matching criteria to provide suggestions by modifying the amount (i.e. a little bit less or more of an item should cause no problems) or replacing it (e.g. chicken instead of beef). When, for example, the amount of each available ingredient is not less than $1 / 2$ of the suggested amount in the recipe description and the total amount of available ingredients is not less than $2 / 3$ of the suggested amount, it can be identified as a possible modification. Furthermore, we could also enhance the set of suggestions by enabling deletion of ingredients. For instance, if one of the ingredients is missing, a recipe could still be suggested by the system. This decision should depend on the importance of the ingredients which can be labeled as critical, somewhat important or supportive. Because of complexity however, we will initially not include these constrains in our first prototype.

\section{B. Expected experiences}

Most participants were enthusiastic about the concept of social recipes, but also noticed disadvantages or detractors. The following reasons were given for using the system, relating clearly to its advantages:

Habit: a number of participants consistently throw away the same type of food as a result of bad predictability about longevity at the time of purchase. A system that could help them in planning their weekly dinners together, using items that have a constant high potential of being wasted, was mentioned to be a solution with high potential.

Awareness: busy lives and forgetting was mentioned to be a main reason of throwing away own and housemates' food. A system that reminds users with their food available at home and its usage potential is perceived as very useful. Especially discounted food items (e.g. economy packages or buy one get one) often end up being forgotten and wasted.

Creativity: some participants were not necessarily only interested in being remembered of what is available but they were also interested in knowing the potential usage of items that did not come to mind initially. The system could help them to realize these possibilities and enhance creativity around cooking.

Surprise: related with creativity, participants from the focus group expect the content as well as the timing of social recipe suggestions to be positive surprises. This would encourage spontaneous meet-ups with fun as a means of motivating behavior change.

Coordination: having a platform that increases users awareness of availability and at the same time supporting the 
coordination for shopping as well as cooking was mentioned to be helpful. This would prevent users that are living together from buying similar or already available items. Coordination is supported by Ganglbauers' [5] visibility dimension for cooperation as having potential to organize daily practices around food and prevent food waste.

The following advantages were given by the focus group. Connectedness: participants expected the proposed system to provide more opportunities for seeing friends. The use of available items from different households together into one recipe and the surprising element of social recipe suggestions were expected to increase the feeling of being connected.

Education: participants also mentioned the potential of social recipe suggestions in supporting the improvement of individual cooking skills. Users could learn from the information provided by the system as well as learn from each other while cooking together. Social recipes could initiate conversations among users when the combination of items is surprising, or when particular items that previously were not planned in dinners can now be used.

Negative feedback: on the other hand, participants also expressed negative attitudes towards the system. The following reasons were given for not using the system.

Preparation values and kitchen constraints; for some participants extensive cooking for others was valued as an individual activity done in advance before the actual dinner as a means of showing hospitality. This preference, however, could also have been influenced by the small space of their kitchens. Other kitchens aspects like a bad working fridge and low quality cooking pans were also mentioned to affect food waste.

Location: location was an indicator of not using the system. Users might prefer going to the grocery store over collaborating with friends due to convenience, when the grocery store is located closer to their homes.

The following disadvantage was given by the focus group. Trust: food is very personal and therefore, according to participants, the use of the system should only be among people that trust each other. Specifically, it was mentioned that users should be able to trust the way others handle food items before they are shared.

We will further continue with the importance of considering location and trust in the design of the social recipes system.

\section{Convenience and importance of location}

The system should consider spatial information. If, for example, a supermarket is located closer than friends' homes, users might find it easier and more convenient to go to the supermarket instead. The system should consider the distance to other users in a group to which a recipe had been suggested. It could also take into account the distances to supermarkets. To increase the attractiveness of a social recipe it could consider ingredients from users who are located not much farther than the closest supermarket or it could minimize the distance to be traveled by all users for each suggested recipe.

1) Implications on the system design: the system should consider the postcodes that are entered in user profiles and suggest accordingly. A constraint value could be defined so that users do not need to travel more than a predefined distance. The optimization function can be defined as:

$$
\forall u \in U: \operatorname{Travel}(u, l) \leq D
$$

where:

- $l$ : is the optimum location.

- $D$ : is the maximum distance.

- $U$ : is the list of users receiving the recipe suggestion.

- Travel $(u, l)$ : gives the distance that user $u$ needs to travel to go to location $l$.

\section{Trust}

From our finding, we can distinguish two types of trust: (1) trust in the suggestions provided by the system and (2) trust in other users that has been suggested with the same social recipe. For the first type of trust, the system could construct user profiles based on what users have bought before (what users like) and provide recipes with familiar food items. Some participants have indicated the importance of receiving suggestions according to the foods they like. Another importance is to consider a balanced diet. The system could provide attractive suggestions for easy-to-make recipes that are nutritionally balanced. Adopting healthy eating patterns are expected to have greater effects on sustainability than just reducing food waste [27]. Furthermore, the system could also include an 'expert' friend-like digital agent that knows how long an item will keep (based on databases of average longevity) and communicates this information to users. This could create a moment of quality evaluation before disposal. This social agent could also prevent users from buying products that are likely to get wasted (based on previous experiences). Persuasive technology research have shown that social feedback by an embodied agent can create behavioral change [18]. Our system could include such an embodied or virtual agent that communicates with users.

For the second type of trust, a parameter can be set for the number of users to suggest social recipes to. This value could be important as it could affect the acceptance rate (i.e. people may enjoy less crowded dinners or it may be more difficult to coordinate with more people). Also, users should have the control in who they would like to connect with through the system for receiving social recipes. Initially in our next studies the groups will be predefined. 
1) Implications on the system design: for now we will only consider optimization functions for the balance of nutrition and the number of users. We can distinguish six classes of nutrients: proteins, fats, carbohydrates, vitamins, minerals and water, but we will only focus on the first three nutritions; the PFC ratio. According to [28], the ideal protein ratio is $10-20$ percent, the ideal fat ratio is $20-25$ percent and the ideal carbohydrate ratio is 50-70 percent depending on age, Basal Metabolic Rate (BMR) and health conditions. For the balance of nutrition, the following optimization function can be defined:

$$
\forall n \in N: \operatorname{Amount}(n) \epsilon\left[n_{\min }, n_{\max }\right]
$$

where:

- $N$ : is proteins, fat and carbohydrates.

- $\operatorname{Amount}(n)$ : is the amount of nutrition $n$.

- $n_{\min }$ : is the minimum nutrition amount somebody should consume for a dinner.

- $n_{\max }$ : is the maximum nutrition amount somebody should consume for a dinner.

For the number of users, which they could manually indicate preferences in the mobile application, the following optimization function can be defined:

$$
\forall r \in R: \text { Number }(r) \leq C
$$

where:

- $R$ : is the list of suggested recipes.

- $C$ : is the optimum number for the users of a recipe.

- Number $(r)$ : gives the number of users who receive recipe $r$.

\section{Discussion AND CONCLUSION}

This paper contributes to the understanding of the relevance and desirability of a community-based social recipe recommendation system, and the design of the system based on user studies. These user studies have shown that there is potential for a community-based social recipe recommendation system which revealed important aspects to be considered for the design of such a system. Its value was found in a variety of aspects such as to compensate for habitual waste-related behavior, awareness, creativity, the triggering of spontaneous actions and surprises, coordination among users, education and the connectivity with friends for social food-related activities, building on food sharing. Based on these findings we discussed possible implications for the design of our community-based social recipe recommendation system.

\section{A. Limitations of the study}

Throughout this process, we ran into limitations of our study. For example, although using receipts as cues in retrospective interviews for reporting wasted foods is a more objective method than surveys, it is still prone to memoryrecall biases. Reported wasted items, for example, are mainly rough estimates. With the mobile application for logging and tracking, we expect to greatly improve the accuracy of measurements and quantification as users can immediately enter the usage or wastage of items after cooking. We are, however, also aware that it is important for users to be motivated to enter this information, which is expected to depend on the perceived value of social recipe suggestions. A solution for this could be to target specific participants in future studies for user and system evaluation. We could, for example, recruit participants from food sharing communities or those who are already sustainable and are therefore interested in using our system. Another possibility is to recruit participants who are already used to using food related applications (e.g. sporters who are tracking their nutrition). People could also be instructed to only enter those items they would not mind sharing. The manual logging of food waste is another limitation, as it might reduce its frequency. Therefore, we are currently working on also automating the measurement of food waste through an augmented bin to weight the waste. This is expected to provide us with more accurate food waste data. Furthermore, the group sizes of our participants are small. We should approach bigger communities of interconnected people with different interpersonal ties. A bigger network of users would correspond to a more realistic setting and could provide different aspects to consider. The challenge is how to target such numbers of users for testing purposes.

\section{B. Future work}

Currently, the optimization functions for the system are open for revisions and changes. Before finalizing the functions and its implementation we will first deploy the mobile application in a second user study with social recipes suggested to users manually to measure its effects on food related behavior. The collected food data will then be used to test the optimization functions in a simulation study which results can be compared with the results derived from the user study. In the user study our main interest lies in how social recipes affect food waste and the social dynamics around food related practices. We will also explore how social recipes affect perception, environmental attitude, social values and general sustainable behavior. The objective of the simulation study is to explore how recipe suggestions could be improved through optimization functions. A system and user evaluation comes with challenges; for example, getting sufficient data. To compensate with small data sets, we are planning on applying Bayesian approaches for data analysis.

\section{ACKNOWLEDGMENT}

We would like to thank all participants for their hospitality and collaboration. This work is supported in part by the Erasmus Mundus Joint Doctorate in Interactive and Cognitive Environments (ICE), which is funded by the EACEA Agency of the European Commission under EMJD ICE FPA n 20100012. 


\section{REFERENCES}

[1] A. Gustavsson, Jenny; Cederberg, Christel; Sonesson, Ulf; Otterdijk van, Robert; Meybeck, "Global food losses and food waste," Food and agriculture organization of the united nations, Tech. Rep., 2011.

[2] W. Moomaw, T. Griffin, K. Kurczak, and J. Lomax, "The critical role of global food consumption patterns in achieving sustainable food systems and food for all," United Nations Environment Programme, Tech. Rep., 2012.

[3] C. Mobley, W. M. Vagias, and S. L. DeWard, "Exploring Additional Determinants of Environmentally Responsible Behavior: The Influence of Environmental Literature and Environmental Attitudes," Environment and Behavior, vol. 42, no. 4, pp. 420-447, Oct. 2009.

[4] T. L. Milfont, J. Duckitt, and L. D. Cameron, "A Cross-Cultural Study of Environmental Motive Concerns and Their Implications for Proenvironmental Behavior," Environment and Behavior, vol. 38, no. 6, pp. 745-767, Nov. 2006.

[5] E. Ganglbauer, G. Fitzpatrick, and G. Molzer, "Creating Visibility : Understanding the Design Space for Food Waste," in Proceedings of the 11th International Conference on Mobile and Ubiquitous Multimedia, 2012, pp. 0-9.

[6] E. V. A. Ganglbauer, G. Fitzpatrick, and R. O. B. Comber, "Negotiating Food Waste : Using a Practice Lens to Inform Design," ACM Transactions on Computer-Human Interaction, vol. 20, no. 2, pp. 1-25, 2013.

[7] A. Olofsson and S. Ohman, "General Beliefs and Environmental Concern: Transatlantic Comparisons," Environment and Behavior, vol. 38, no. 6, pp. 768-790, Nov. 2006.

[8] R. Comber, J. Hoonhout, A. van Halteren, P. Moynihan, and P. Olivier, "Food Practices as Situated Action : Exploring and designing for everyday food practices with households," in Proceedings of the SIGCHI Conference on Human Factors in Computing Systems, 2013, pp. 24572466.

[9] E. Ganglbauer, W. Reitberger, and G. Fitzpatrick, "An Activist Lens for Sustainability : From Changing Individuals to Changing the Environment," in Persuasive, 2013, pp. 63-68.

[10] V. Lim, F. Yalvac, M. Funk, J. Hu, and M. Rauterberg, "Can we reduce waste and waist together through EUPHORIA ?" in The Third IEEE International Workshop on Social Implications of Pervasive Computing, 2014, pp. 382-387.

[11] H. Brynjarsdóttir, M. Hå kansson, J. Pierce, E. P. S. Baumer, C. Disalvo, and P. Sengers, "Sustainably Unpersuaded : How Persuasion Narrows Our Vision of Sustainability," in Proceedings of the SIGCHI Conference on Human Factors in Computing Systems, 2012, pp. 947-956.

[12] J. Froehlich, L. Findlater, and J. Landay, "The Design of Eco-Feedback Technology," in Proceedings of the SIGCHI Conference on Human Factors in Computing Systems, 2010, pp. 1999-2008.

[13] J. Pierce, W. Odom, and E. Blevis, "Energy Aware Dwelling : A Critical Survey of Interaction Design for Eco-Visualizations," in Proceedings of the 20th Australasian Conference on Computer-Human Interaction, 2008, pp. $1-8$.

[14] T. Holmes, "Eco-visualization : Combining art and technology to reduce energy consumption," in ACM SIGCHI conference on Creativity \& cognition, 2007, pp. 153-162.
[15] J. Froehlich, S. Patel, J. a. Landay, L. Findlater, M. Ostergren, S. Ramanathan, J. Peterson, I. Wragg, E. Larson, F. Fu, and M. Bai, "The design and evaluation of prototype eco-feedback displays for fixture-level water usage data," in Proceedings of the SIGCHI Conference on Human Factors in Computing Systems. New York, New York, USA: ACM Press, 2012, pp. 2367-2376. [Online]. Available: http://dl.acm.org/citation.cfm?doid=2207676.2208397

[16] J. Maitland, M. Chalmers, and K. A. Siek, "Persuasion not Required Improving our Understanding of the Sociotechnical Context of Dietary Behavioural Change," in PervasiveHealth, no. Cvd, 2009, pp. 1-8.

[17] S. Foster, Derek; Lawson, "Liking Persuasion : Case studies in Social Media for Behaviour Change," in Extended Abstracts on Human Factors in Computing Systems, 2013, pp. 1-8.

[18] C. Midden and J. Ham, "Chapter 23 Persuasive technology to promote environmental behaviour," in Environmental Psychology; an introduction, 2013, pp. 243-254.

[19] A. Grimes and R. Harper, "Celebratory Technology : New Directions for Food Research in HCI," in Proceedings of the SIGCHI Conference on Human Factors in Computing Systems, 2008, pp. 467-476.

[20] S. Gross, A. Toombs, J. Wain, and K. Walorski, "Foodmunity : Designing community interactions over food," in Extended Abstracts on Human Factors in Computing Systems, Gross2011, 2011, pp. 1019-1024.

[21] M. Svensson, K. Hook, and R. Coster, "Designing and Evaluating Kalas : A Social Navigation System for Food Recipes," ACM Transactions on Computer-Human Interaction, vol. 12, no. 3, pp. 374-400, 2005.

[22] A. Grimes, M. Bednar, J. D. Bolter, R. E. Grinter, and C. S. Atlanta, "EatWell : Sharing Nutrition-Related Memories in a Low-Income Community," in ACM Conference on Computer-Supported Cooperative Work and Social Computing, 2008, pp. 87-96.

[23] P. Barden, R. Comber, D. Green, D. Jackson, C. Ladha, T. Bartindale, N. Bryan-kinns, T. Stockman, and P. Olivier, "Telematic Dinner Party : Designing for Togetherness through Play and Performance," in Proceedings of the Designing Interactive Systems Conference, 2012, pp. $38-47$.

[24] H. Kanai and K. Kitahara, "A Menu-planning Support System to Facilitate Communication among Neighbors," in ACM Conference on Computer-Supported Cooperative Work and Social Computing, 2011, pp. 661-664.

[25] E. Ganglbauer, G. Fitzpatrick, O. Subasi, and F. Güldenpfennig, "Think Globally , Act Locally : A Case Study of a Free Food Sharing Community and Social Networking," in ACM Conference on ComputerSupported Cooperative Work and Social Computing, 2014, pp. 911-921.

[26] G. Liu, X. Liu, and S. Cheng, "Food security: Curb China's rising food wastage," Nature, vol. 498, p. 170, 2013.

[27] M. M. Rutten, "Reducing food waste by households and in retail in the eu: A prioritisation on the basis of economic, land use and food security impacts," in Proceedings of the First International Conference on Food Security, 2013, noordwijkerhout, Netherlands.

[28] C. Nishikawa, A. Nagai, T. Ito, and S. Maruyama, "Contemporary Challenges and Solutions in Applied Artificial Intelligence," vol. 489, pp. 55-60, 2013. [Online]. Available: http://link.springer.com/10.1007/9783-319-00651-2 